\title{
Die Herstellung von Dauerpräparaten aus Harnsedimenten.
}

Von Elise Wolff, Präparatorin am Privatlaboratorium von Prof. A. Fraenkel in Berlin.

Färbungen und Konservierungen von Harnsedimenten sind schon vielfach gemacht worden. Auch die Anfertigung von Danerpräparaten wurde versucht; doch sind in der einschlägigen Literatır Angaben iiber gut gefärbte, lange haltbare Präparate, wie sie in der histologischen Technik erzielt werden und welche fur Kurse. Demonstrations und forensische Zwecke, als Kontroll- und Belegpräparate bei chronischem Krankheitsverlauf wertvoll sind, nicht zu finden.

Die Autoren, wie Kuttner (1), Senator (2), Fischel (3), Rohnstein (4), Martin Cohn (5). beschränkten sich entweder auf Fettreaktionen mit eventueller Nachfärbung und Aufbewahrıng in Glyzerin oder ließen das Zentrifugat auf dem Deckglas antrocknen und färbten dann. Die Glyzerinaufbewahrung bedarf aber der Kontrolle. da bei nicht völligem Luitabschluß leicht eine Zersetzung stattfinden kann; ferner wird das ungefärbte Material zu durchsichtig. die Konturen der Formbestandteile verwischen sich z.u sehr. und zu etwaiger späterer Nachfärbung ist wieder eine völlige Entfernung des Glyzerins nur durch gründliches Auswaschen möglich, wodurch eine Schadigung des Materials nicht vermieden werden kann. Deckglasausstriche geben auch kein klares, instruktives Bild. Die Formelemente, besonders die so zarten hyalinen Zylinder sehen oft destruiert, jedenfalls stets verändert aus.

Schon seit längerer Zeit hatte auch ich mich mit Versuchen beschäftigt, wirkliche Dauerpräparate, in welchen die Formbestandteile gut erhalten und gefärbt sind, herzustellen. Yon einer schon im Jahre 1896 erschienenen, leider wenig bekannten Arbeit Gump rechts (6) über denselben Gegenstand erhielt ich erst Kienntnis, als meine Versuche, welche ich zuerst fast in derselben Weise gemacht hatte, beinahe abgeschlossen walen. Allerdings $\mathrm{kam}$ ich durch eine Arbeit Heidenhains (7), welcher die Zentrifuge zur Gewinnung von Präparaten isolierter Zellen zu Kurszwecken anwendete, auf die Idee, dieses Verfahren. wenn auch modifiziert, für Harnsedimente zu verwerten. Die Erfahrung lehırte mich aber, da $\beta$ die Zentrifuge, ausschließlich angewendet, entgegen der $\mathrm{G}$ umprechtschen Ansicht doch schädigend wirkt. Außerdem ist gerade bei sedimentarmen Harnen, bei welchen wiederholtes Zentrifugieren und Dekantieren zur Erlangung eines genügenden Sedinentes notwendig ist, der Zeit- und Kraftaufwand so groß, besonders wenn man auf eine Handzentrifuge angewiesen ist, da $B$ das Fertigen vun Dauerpräparaten sich sehr milhsam gestalten wiirde. Fur die Schnelluntersuchung am ungefärbten Präparat ist die Zentrifuge nicht zu entbehren, zur Anfertigung von Dauerpräparaten kann ihre Anwendung fast ganz eingeschränkt werden. weil besondere Eile dabei nicht nötig ist. Der Gang der Präparation kann wie bei histologischen Untersuchungen unterbrochen und wieder aufgenommen werden.

Zur Fixation, nachdem ich die verschiedensten Fixationsmittel probiert hatte, haben sich mir am besten $10 \%$ iges Formol allein oder mit Nachfixierung von $90 \%$ igem Alkohol oder alleinige Fixierung mit letzterem bewährt. Stärkerer Alkohol wirkt destruierend, schwächerer fixiert nicht genügend. Con Methylalkohol. den ich auch probierte, sah ich keine besonderen Vorteile. Müllersche Flüssigkeit (Bohland [8]) ist nicht zweckmäfig. da sie zu langsam fixiert und vielfaches Auswaschen vellangt. Sublimat fixiert zwar schnell, beansprucht aber haufiges Auswaschen, eventuell auch mit jodhaltigem Alkohol, da stórende Niederschläge entstehen, wenn es nicht völlig entfernt ist.

Das Verfahren gestaltet sich in folgender Weise:

Wenn sich nach kurzem Zentrifugieren oder Stehenlassen in hohem Spitzglase genügendes Sediment absetzt, wird der überstehende Harn abgegossen und die Fixationsflüssigkeit aufgefüllt. andernfalls wird im Spitzglase der zu velarbeitende Harn mit dem Fixierungsmittel im Verhältnis von etwa 1:5 gemischt. Alkohol allein ist notwendig, wenn es sich um Fixierung roter Blutkörperchen handelt Ganz entgegen der Erfahrung an histologischen Präparaten, 
in welchen gerade Formol blutfixierend wirkt, laugt dasselbe, wie auch Gumprecht beobachtet hat, im Harn die Blutkörperchen aus, während Alkohol sie ausgezeichnet erhält. Mit Eosin färben sie sich dann leuchtend rotgelb. Vorheriges Auswaschen mit Wasser oder physiologischer Kochsalzlösung ist nicht zu empfehlen; sie wirken zwar lösend auf Urate, was ja Formol auch tut, doch ungünstig, da sie nicht fixieren, auf die Formbestandteile.

Die reichliche Fixationsflüssigkeit mischt sich schnell und gieichmäßig mit dem Sediment, resp. dem Harn, bewirkt eine schnelle und gleichmäßige Fixierung und verhindert jede Zersetzung. Das Glas muß jetzt sowie auch bei den nachfolgenden Manipulationen verdeckt gehalten werden, um Eindringen von Staub etc. sowie das Verdunsten des Alkohols zu verhindern. Am nächsten Tage hat sich starkes Sediment abgesetzt. Natïrlich werden je nach der Art der Fixierung Salze, Eiweiß etc. mit ausgefällt, aber dadurch werden auch gerade bei Harnen mit spärlichen Formbestandteilen diese mit niedergerissen, und infolgedessen wird ihr Auffinden erleichtert. Die überstehende Flüssigkeit wird abgegossen, das Formol durch Alkohol ersetzt, resp. letzterer gewechselt. Das aufgewirbelte Sediment setzt sich jetzt sehr schnell ab, meist schon in 10-15 Minuten, dann Abgießen des Alkohols und eventuell für Hämatoxylinfärbung - unbedingt nötig ist es nicht - Auffüllen von destilliertem Wasser. Wenn sich das Sediment zu sehr zusammenballt, so muß es leicht aufgeschüttelt oder mit einem Glasstab vorsichtig umgerihrt werden. Jetzt wird das Sediment in die Zentrifugenröhre gespült, das Wasser, resp. der Alkohol abgegossen, die Färbeflüssigkeit hineintiltriert. Zur Erbaltung der Kristalle nach Alkoholfixierung muß eine alkoholische Farbstofflösung genommen werden. Die besten, instruktivsten Bilder gibt aber die Doppelfärbung mit Hämatoxylin-Eosin. Langsame Färbung, 24 Stunden mit verdünnter Lösung, ist am empfehlenswertesten. Die Durchfärbung ist eine sehr gleichmäßige, eine Ueberfärbung ausgeschlossen. Selbstverständlich läßt sich unter mikroskopischer Kontrolle die Färbung mit konzentrierterer Lösung auch ausführen. Das Sediment ist aber in der dunklen Flüssigkeit schwer zu sehen, beim Abgießen muB sehr vorsichtig verfahren werden, damit nicht zuviel davon verloren geht. Allerdings ist zur Schnellfärbung eine viel geringere Menge der Farblösung nötig; man hilft sich, indem man sie nicht abgießt, sondern später destilliertes Wasser zufiullt. Dann ist die Beobachtung leichter. Das Sediment pflegt sich auch jetzt sehr schnell abzusetzen, ein Zentrifugieren ist bei all diesen Manipulationen fast nie notwendig. Abgießen des blau gefärbten Wassers, resp. des Hämatoxylins, Auffüllen von Leitungswasser, nach Absetzen Wechseln desselben, bis es nicht mehr gefärbt erscheint (zweimal genügen meistens) und, wenn möglich, Stehenlassen des zugepfropften Röhrchens bis zum nächsten Tage. Je länger die Wässerung, desto besser wird die Färbung. Dann Eintropfen von einigen, etwa 5-6, Tropfen konzentrierter alkoholischer Eosinlösung und nach einigen Minuten Zufüllen von $90 \%$ igem Alkohol, in welchem sich die Differenzierung vollzieht. Das Sediment kann jetzt sowie auch vor der Färbung in Alkohol beliebig lange aufbewahrt werden. Man muß nur dafür sorgen, daß durch guten Verschluß der Röhrchen die Verdunstung des Alkohols und eine Eintrocknung verhindert wird. Zur Fertigstellung des Präparates entnimmt man mit der Pipette einen Tropfen des Sedimentes, tut ihn auf den Objektträger und kontrolliert mikroskopisch Färbung und Differenzierung. Genügt beides, so wird der überschüssige Alkohol durch Absaugen mittels Fließpapiers entfernt. Dann von der Seite Hinzufließenlassen einiger Tropfen absoluten Alkohols, Absaugen desselben, noch einmal absoluter Alkohol, Entfernen desselben, dann auch von der Seite Hinzutun einiger Tropfen Karbol - oder reinen Xylols, Absaugen, neutraler Balsam, resp. Zedernöl, das auch fest wird und in welchem sich alle Färbungen ganz vorzüglich halten.

Zum Gelingen des Präparates ist es aber unbedingt erforderlich, dab das Sediment auf dem Objektträger währendaller Manipulationen feucht bleibt; es darf bei dem Wechseln des Alkohols nie trocken werden; ferner muB die Entwasserung eine rollständige sein, soda $B$ beim Hinzufügen des Xylols der Sedimenttropfen nicht trübe wird. Beachtet man diese MaBregeln nicht, so ist das Präparat unrettbar verdorben; Zurückgehen zum Alkohol. nochmaliges Entwässern nützen nichts; das Bild wird unklar, unsauber, die Konturen der Zellen werden verwischt, die $\mathbf{Z y -}$ linder destruiert.

Bei vorsichtigem Manipulieren schwimmt der Tropfen des Sedimentes trotz des mehrmaligen Flüssigkeitswechsels nicht fort, er breitet sich meist nur etwas mehr aus, was durchaus kein Fehler ist, da die einzelnen Formbestandteile dadurch isolierter zur Darstellung gelangen. Sind sie sehr reichlich, sind wenig, resp. keine gefallten Salze etc. vorhanden, dann haftet das Sediment sogar ganz fest, wohl infolge von Capillarattraktion, auf dem Objekttrager. Die mitfixierten und auch gefärbten andern Bestandteile, wie Salze, Eiweiß, machen das mikroskopische Bild bei der angegebenen $\mathrm{Be}$ handlung durchaus nicht unklar oder unsauber. Zylinder, Zellen etc. heben sich prägnant ab. Liegen sie bei sehr reichlichem Sediment zu dicht beieinander, so kann man, wenn das Deckglas aufgelegt ist, ganz leicht ein wenig mit der Nadel aufdrücken, wodurch der Tropfen auseinanderfließt und die Uebersicht eine klare wird.

Für van Gieson-Färbung muß eine sehr starke Ueberfärbung mit Hämatoxylin stattgefunden haben, sodaß die Differenzierung so lange ausgedehnt werden kann, bis sich mittels Zentrifugieren oder durch Sedimentieren ein genügender Satz gebildet hat. Dann Abgießen der Mischung, Auffüllen von mit Pikrin versetztem 90\% igem Alkohol und Weiter wie oben. Das Aufbewahren des Sedimentes in Xylol, wie Heidenhain es tut, halte ich nicht für ratsam, da lange Einwirkung von Xylol schrumpfend, ev. auch entfärbend wirkt.

Karminfärbungen sind sehr gut anwendbar. Nach Abgießen des 90\% igen Alkohols Versetzen des Sedimentes mit dem betreffenden Karmin. Nach erfolgter Färbung, die von $1 / 2$ Stunde an, beliebig lange ausgedehnt werden kann, Abgießen der Farblösung, Wasserspülung, dann Aufgießen von $1 \%$ igem salzsauren Alkohol, welcher ebenso wie das Wasser und später der absolute Alkohol und das Xylol nach Pikrokarminfärbung mit Pikrin versetzt werden muß. Differenzierung bis zum nächsten Tage. Dann erst $90 \%$ iger Alkohol zum Entfernen des salzsauren und weiter, wie früher angegeben. Färbung nach Giemsa läßt sich natürlich auch ausführen.

Am SchluB dieser Arbeit erhielt ich noch Kenntnis von einem von Fiorentini und Signer (9) veröffentlichten Artikel über Färbung und Konsurvierung von Harnsedimenten. Die Verfasser färben das Zentrifugat ohne Fixierung mit Triacid und differenzieren und konservieren in leicht saurem Glyzerin. Die Resultate sollen sehr gute sein und die Präparate sich monatelang halten.

Literatur: 1. Kuttner. - 2. Senator, Virchows Archiv, Bd. 131 und Enzy. klopādie der mikroskopischen Technik, S, 520-522 - 3. F is chel, Prager medizinische Wochenschrift 1895 . Referat des Zentralblattes für innere Medizin 1895. 4. Rohnstein, Fortschritte der Medizin 1902. - 5. Martin Cohn, Zeitschrift fur

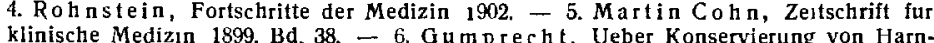
klinische Medizın 1899, Bd. 38. - 6. Gumprecht, Ueber Konservierung von Harn-
sedimentell. Zentralblatt tür innere Medizin 1896, No. 30, S. $761 .-7$. Heide nh a in

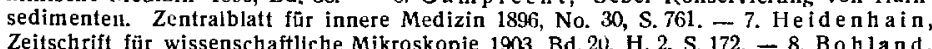
Zeitschrift für wissenschaftliche Mikroskopie 1903, Bd. 21 , H. 2 , S. $172,-8$. Bo hland,
Zentralblatt fdr innere Medizin 1894, No. $20-9$. Pietro Fiorentini und M. Sig. ner, Zetschrift für wissenschaftliche Mikroskople 1905, Bd. 22, H. 2, S. $18 \bar{t}$ 